\title{
Cosmological test using the Hubble diagram of high- $z$ quasars
}

\author{
Fulvio Melia ${ }^{\oplus \star} \dagger$ \\ Department of Physics, The Applied Math Program, and Department of Astronomy, The University of Arizona, Tucson, AZ 85721, USA
}

Accepted 2019 July 26. Received 2019 June 9; in original form 2019 March 8

\begin{abstract}
It has been known for over three decades that the monochromatic X-ray and UV luminosities in quasars are correlated, though non-linearly. This offers the possibility of using high- $z$ quasars as standard candles for cosmological testing. In this paper, we use a recently assembled, high-quality catalogue of 1598 quasars extending all the way to redshift $\sim 6$, to compare the predictions of the $R_{\mathrm{h}}=c t$ and Lambda cold dark matter $(\Lambda \mathrm{CDM})$ cosmologies. In so doing, we affirm that the parameters characterizing the correlation depend only weakly on the chosen cosmology, and that both models account very well for the data. Unlike $\Lambda$ CDM, however, the $R_{\mathrm{h}}=c t$ model has no free parameters for this work, so the Bayesian Information Criterion favours it over $\Lambda \mathrm{CDM}$ with a relative likelihood of $\sim 88$ per cent versus $\sim 10$ per cent. This result is consistent with the outcome of other comparative tests, many of which have shown that $R_{\mathrm{h}}=c t$ is favoured over the standard model based on a diverse range of observations.
\end{abstract}

Key words: quasars: supermassive black holes - cosmological parameters - cosmology: distance scale-cosmology: observations - cosmology: theory.

\section{INTRODUCTION}

The discovery of quasars at redshifts $z>5-6$ (Fan et al. 2003; Jiang et al. 2007; Willott et al. 2007; Mortlock et al. 2011; Banados et al. 2014) represents - on the one hand - an enduring mystery in astronomy given that the emergence of $10^{9-10} \mathrm{M}_{\odot}$ supermassive black holes only $\sim 900 \mathrm{Myr}$ after the big bang and, worse, only $\sim 500 \mathrm{Myr}$ after their likely seeding in Pop II stellar explosions (Melia 2013a) is inconsistent with the timeline in Lambda cold dark matter $(\Lambda \mathrm{CDM})$. To address this problem, one needs to invoke either an anomalously high accretion rate (Volonteri \& Rees 2006) or the generation of unusually massive seeds (Yoo \& Miralda-Escudé 2004). But neither of these effects has ever been observed. For example, Willott et al. (2010) found that no known high- $z$ quasar accretes at more than 1-2 times the Eddington limit (see also Melia 2014; Melia \& McClintock 2015).

On the other hand, these bright objects are being studied at redshifts well beyond the current reach of Type Ia SN observations, which tend to be restricted to redshifts $\lesssim 2$ (see e.g. Jones et al. 2013). They therefore provide a crucial probe of the geometry of the Universe at luminosity distances not easily accessible by other means. In recent years, a Hubble diagram of high- $z$ quasars has been constructed using various techniques for the identification of standard candles, though with variable success depending, in large part, on the quality and size of the sample.

\footnotetext{
^E-mail: fmelia@email.arizona.edu

$\dagger$ John Woodruff Simpson Fellow.
}

For example, the Mg II full width at half-maximum (FWHM) and UV luminosity of quasars at $z>6$ appear to be correlated, and since reverberation mapping of their broad lines can at once yield the optical/UV luminosity and the distance from the central ionizing source to the line-emitting gas, these observations allow us to measure the black hole mass (Blandford \& McKee 1982; Wandel, Peterson \& Malkan 1999; Kaspi et al. 2000; Shen, Greene \& Strauss 2008; Bentz et al. 2009; Steinhardt \& Elvis 2010). And since these high- $z$ quasars also appear to be accreting near their Eddington limit, these measurements may thus be used to construct a Hubble diagram. Unfortunately, the sample ( $\sim 20$ sources $)$ available for this kind of work is still modest, so the outcome of this analysis is suggestive, though not yet conclusive (Melia 2014). None the less, the analysis based on this technique showed, in a one-onone comparison, that the Friedmann-Lemaitre-Robertson-Walker (FLRW) cosmology known as the $R_{\mathrm{h}}=c t$ universe (Melia 2003, 2007, 2013b; Melia \& Shevchuk 2012) is preferred by these data over the standard model $\Lambda$ CDM. Statistical tools, such as the 'Akaike' (Akaike 1973), 'Kullback,' and 'Bayes' (Schwarz 1978) Information Criteria, all demonstrated that the former model is favoured over the latter with a likelihood of $\sim 85$ per cent versus $\sim$ 15 per cent. An analogous study using a sample of 35 active galactic nuclei at lower redshifts produced very similar, confirming results (Melia 2015b).

A very different technique, based on the flat spectrum radio quasar (FSRQ) gamma-ray luminosity function (Zeng, Melia \& Zhang 2016), produced much stronger conclusions, mainly because of the much bigger sample of such sources assembled with Fermi during its 4-yr survey. Using a Kolmogorov-Smirnov test on one-parameter cumulative distributions in luminosity, redshift, photon index, and 
source count, this study concluded that $R_{\mathrm{h}}=c t$ is very strongly favoured over $\Lambda \mathrm{CDM}$.

A third approach (López-Corredoira et al. 2016), much more closely aligned with the work reported in this paper, uses a recently refined method of sampling the redshift-distance relationship (Risaliti \& Lusso 2015), based on a correlation between the X-ray and UV monochromatic luminosities of quasars, first discussed in this context over three decades ago by Avni \& Tananbaum (1986). The fact that this relationship is seemingly independent of evolution may render this method the best used with quasars thus far. In the comparative analysis of nine different cosmological models carried out by López-Corredoira et al. (2016), only two showed very strong consistency with these quasar data, while several others, including Einstein-de Sitter, the Milne universe and static universe models based on tired light, were excluded at $>99$ per cent confidence level.

In this paper, we revisit the X-ray versus UV luminosity correlation for quasars, in light of the much improved sample of suitable sources assembled from the literature and follow-up observations by Risaliti \& Lusso (2019). These data are interpreted in the context of a generally accepted scenario, in which the UV photons are emitted by an accretion disc, while the X-rays are Compton upscattered photons from an overlying, hot corona. But though a non-linear correlation to link these two spectral features has been known for many years, only recently has an uncomfortably large dispersion in the relation been mitigated with a more refined selection of the sources. Most of the quasars in the parent sample have been identified from the crosscorrelation of the XMM-Newton Serendipitous Source Catalogue Data Release 7 (Rosen et al. 2016) with the Sloan Digital Sky Survey (SDSS) quasar catalogues from Data Release 7 (Shen et al. 2011) and 12 (Paris et al. 2017). With the aim of whittling this catalogue down to those sources with reliable measurements of the intrinsic X-ray and UV emissions, avoiding possible contaminants and unknown systematics, Risaliti \& Lusso (2019) produced a final, high-quality catalogue of 1598 quasars that we shall use for the analysis reported in this paper.

In Section 2, we introduce the two cosmological models we shall compare in this study, along with a 'cosmographic' empirical fit used by Risaliti \& Lusso (2019) to provide a less-model-dependent analysis of the quasar data. Ironically, this approach ends up requiring more unknown parameters for the fit than the models themselves, so it actually does not fare better than the latter when used with Information Criteria. We discuss the results of our model comparison in Section 3, and conclude in Section 4.

\section{METHODOLOGY}

The non-linear relation between the quasar's UV (disc) and X-ray (coronal) emissions is usually parametrized as

$\log _{10} L_{\mathrm{X}}=\gamma \log _{10} L_{\mathrm{UV}}+\beta$,

where $L_{\mathrm{X}}$ and $L_{\mathrm{UV}}$ are the rest-frame monochromatic luminosities at $2 \mathrm{keV}$ and $2500 \AA$, respectively. The logarithms in this expression may be taken in any base, though we restrict our usage to base 10 throughout this paper. Previous studies have shown that $\gamma \sim$ 0.5-0.7 (Avni \& Tananbaum 1986; Just et al. 2007; Lusso et al. 2010; Young, Risaliti \& Elvis 2010; Risaliti \& Lusso 2015; Lusso \& Risaliti 2016; Risaliti \& Lusso 2019). Since the data contain the fluxes, rather than the absolute (model-dependent) luminosities, we use equation (1) in the modified form

$\log _{10} F_{\mathrm{X}}=\tilde{\beta}+\gamma \log _{10} F_{\mathrm{UV}}+2(\gamma-1) \log _{10} d_{\mathrm{L}}$, where $\tilde{\beta}$ is a constant that subsumes the slope $\gamma$ and the intercept $\beta$ in equation (1), such that

$\tilde{\beta}=\beta+(\gamma-1) \log _{10} 4 \pi$.

For each model, we optimize its parameters (if it has any), along with those characterizing the empirical fit in equation (2), i.e. $\gamma, \widetilde{\beta}$, and $\delta$ (see below), by minimizing the likelihood function

$\ln (L F)=-\sum_{i=1}^{1598}\left\{\frac{\left[\log _{10}\left(F_{\mathrm{X}}\right)_{i}-\Phi\left(\left[F_{\mathrm{UV}}\right]_{i}, d_{\mathrm{L}}\left[z_{i}\right]\right)\right]^{2}}{\tilde{\sigma}_{i}^{2}}+\ln \left(\tilde{\sigma}_{i}^{2}\right)\right\}$,

where the variance $\tilde{\sigma}_{i}^{2} \equiv \delta^{2}+\sigma_{i}^{2}$ is given in terms of the global intrinsic dispersion, $\delta$, and the measurement error $\sigma_{i}$ in $\left(F_{\mathrm{X}}\right)_{i}$ (Risaliti \& Lusso 2015). The error in $\left(F_{\mathrm{UV}}\right)_{i}$ is presumed to be insignificant compared to $\sigma_{i}$ and $\delta$, and is therefore ignored in this application. In equation (4), the function $\Phi$ is defined as

$\Phi\left(\left[F_{\mathrm{UV}}\right]_{i}, d_{\mathrm{L}}\left[z_{i}\right]\right) \equiv \tilde{\beta}+\gamma \log _{10}\left(F_{\mathrm{UV}}\right)_{i}+2(\gamma-1) \log _{10} d_{\mathrm{L}}\left(z_{i}\right)$,

in terms of the measured fluxes $\left(F_{\mathrm{X}}\right)_{i}$ and $\left(F_{\mathrm{UV}}\right)_{i}$ at redshift $z_{i}$.

The three models we compare here are (i) flat $\Lambda \mathrm{CDM}$, (ii) the $R_{\mathrm{h}}=c t$ universe, and (iii) a cosmographic (empirical) fit using a low-order polynomial for the luminosity distance. We have included $R_{\mathrm{h}}=c t$ because a variety of previously published comparative tests between this model and $\Lambda \mathrm{CDM}$ have shown that it accounts for the data as well, if not better, than the standard model. Some counter claims have also been published in recent years, and we summarize some of these here to demonstrate that the issue of whether or not $R_{\mathrm{h}}=c t$ is the correct cosmology still needs to be resolved. The analysis in this paper advances this discussion significantly by providing an important new comparison between these models using observations over an unprecedentedly large range in redshifts (see Fig. 5 below).

Over the past decade, $R_{\mathrm{h}}=c t$ has been compared to $\Lambda \mathrm{CDM}$ using data at low, high, and intermediate redshifts, based on a variety of integrated observational signatures, such as the luminosity and angular-diameter distances, and also differential measures, such as the redshift-dependent Hubble parameter. A very helpful summary of these analyses and their outcomes appears in table 2 of Melia (2018a). Some of these data, however, are often subject to unknown systematics and, worse, are themselves sometimes dependent on the assumed background model. A well-known example is the analysis of Type Ia SNe, whose light curve is characterized by three or four so-called 'nuisance' parameters that must be optimized along with the unknown variables in the cosmological model. Depending on the assumptions made (e.g. with regard to unknown intrinsic dispersions) and techniques used (e.g. $\chi^{2}$ minimization versus the maximization of the likelihood function and its relation to information criteria), the outcome of these tests can sometimes change from one application to another.

So in addition to the results reported in table 2 of Melia (2018a), some counter claims have also been published, questioning whether $R_{\mathrm{h}}=c t$ is in fact favoured by the data over $\Lambda \mathrm{CDM}$. This is the principal reason why additional comparative tests, such as that presented in this paper, are critical to this ongoing discussion. For example, the use of Type Ia SNe for model testing is especially difficult when merging disparate subsamples to improve the statistics, since each comes with its own unknown systematics. Shafer (2015) combined the Union2.1 and JLA samples for this purpose, and concluded that these data favour the standard model. But in his analysis, he avoided having to deal with the unknown intrinsic dispersions by constraining the reduced $\chi^{2}$ of each subsample to be 1 . A better statistical approach (Kim 2011; Wei et al. 2015; Melia et al. 
2018) estimates these unknowns by instead maximizing the overall likelihood function. Depending on which of these assumptions and methods one adopts, the outcome of which model is preferred by the SN data changes.

A more recent example is the use of local probes for model testing (Lin, Li \& Sang 2018), in which SN data were combined with measurements of $H(z)$ and baryon acoustic oscillations (BAOs). These authors also concluded that $\Lambda \mathrm{CDM}$ is favoured by these observations, in contrast to other work in which the opposite result was reported (see e.g. Melia \& López-Corredoira 2017; Melia et al. 2018). The difference can be easily traced to which data sets were used in the two studies. Like the SN measurements, the BAOs themselves generally do not provide model-independent information because the BAO peak location cannot be disentangled from redshift space distortions. To date, only three or four BAO measurements have provided an unambiguous peak location. Thus, any use of BAO data, and measurements of $H(z)$ derived from them, yields a biased outcome. Lin et al. (2018) used all of the data and, not surprisingly, concluded that $\Lambda \mathrm{CDM}$ is favoured, because the standard model was assumed as the background in order to estimate the redshift space distortions. But when only the model-independent data are used (see e.g. Melia \& López-Corredoira 2017) one reaches the opposite conclusion.

This is a principal reason why our model-independent approach in this paper is indispensable. To fully utilize the formalism developed in equations (1)-(5), we need the luminosity distances in the three models we consider here, which are given as

$d_{\mathrm{L}}^{R_{\mathrm{h}}=c t}(z)=\frac{c}{H_{0}}(1+z) \ln (1+z) ;$

$d_{\mathrm{L}}^{\Lambda \mathrm{CDM}}=\frac{c}{H_{0}}(1+z) \int_{0}^{z} \frac{d u}{\sqrt{\Omega_{\mathrm{m}}(1+u)^{3}+\Omega_{\Lambda}}}$

for the minimal one-parameter flat concordance model, in which $\Omega_{\mathrm{m}}$ is the scaled matter density today and the cosmological constant energy density is $\Omega_{\Lambda}=1-\Omega_{\mathrm{m}}$, and

$d_{\mathrm{L}}^{\cos }=\ln (10) \frac{c}{H_{0}}\left[\log _{10}(1+z)+a_{2} \log _{10}^{2}(1+z)+a_{3} \log _{10}^{3}(1+z)\right]$

for the 'cosmographic' empirical fit, based on a third-order polynomial with two constants, $a_{2}$ and $a_{3}$, that need to be optimized along with the other free parameters (see Risaliti \& Lusso 2019).

The Hubble constant $H_{0}$ is not independent of $\tilde{\beta}$, and is subsumed into this parameter during the optimization procedure. To make the figures easy to interpret, however, particularly the Hubble diagram, we assume a fiducial value $H_{0}=70 \mathrm{~km} \mathrm{~s}^{-1}$ $\mathrm{Mpc}^{-1}$ throughout this paper. If one prefers a different Hubble parameter, say $H_{0}^{\prime}$ then, according to equation (2), the optimized values of $\tilde{\beta}$ in Table 1 simply need to be changed by an amount $\Delta \tilde{\beta}=2(\gamma-1) \log _{10}\left(H_{0}^{\prime} / H_{0}\right)$. Notice, therefore, that in this model comparison, the $R_{\mathrm{h}}=c t$ cosmology has no free parameters, $\Lambda \mathrm{CDM}$ has one, and the cosmographic fit has two. This difference is crucial when evaluating the Bayesian Information Criterion (BIC) for the comparison of the model fits, as we shall see shortly.

When models being compared have different numbers of free parameters, as is the case for $R_{\mathrm{h}}=c t$ and $\Lambda \mathrm{CDM}$, a simple $\chi^{2}$ minimization, or even a comparison of likelihood functions, is not sufficient to fairly decide which cosmology is a better match to the data. The sample we have here is very large, and therefore the most appropriate model selection tool to use is the Bayes Information Criterion (Melia \& Maier 2013), which approximates the computation of the (logarithm of the) 'Bayes factor' for deciding between models (Schwarz 1978; Kass \& Raftery 1995). In general terms, information criteria such as this may be viewed as enhanced 'goodness-of-fit' tests, extending the better known $\chi^{2}$ criterion by incorporating the number of model parameters. Information criteria penalize models with a larger number of unknowns, unless they yield a substantially better fit to the data. This enhancement reduces the possibility of overfitting, arising from the fact that, with more parameters, one may simply be adjusting to the noise.

For each model being tested, the BIC is defined by

$\exp (-\mathrm{BIC} / 2) \equiv n^{-k / 2} L^{*}$,

where $L^{*}=e^{-\ln (L F)}$ is the maximized likelihood (from equation 4), $n$ (=1598 here) the data set size, and $k$ the count of free parameters in the model. Then, the relative likelihood of model $\alpha$ being correct is the Bayes probability

$P(\alpha)=\frac{\exp \left(-\mathrm{BIC}_{\alpha} / 2\right)}{\sum_{\beta} \exp \left(-\mathrm{BIC}_{\beta} / 2\right)}$,

where $\exp \left(-\mathrm{BIC}_{\alpha} / 2\right)$ is its 'Bayes weight'. The Bayesian interpretation of this method is as follows: $\exp \left(-\mathrm{BIC}_{\alpha} / 2\right)$ is a large-sample $(n \rightarrow \infty)$ approximation to an integral over the parameter space of model $\alpha$, of its likelihood function $L$. The standard error of each parameter shrinks like $n^{-1 / 2}$ in this limit.

These probabilities are, of course, relative ones so, in principle, the ratio of any two of them represents the factor by which either model is more likely to be 'closer to the correct' cosmology. For the $\mathrm{BIC}$, there is an accepted interpretation of the magnitude of the difference $\Delta=\mathrm{BIC}_{2}-\mathrm{BIC}_{1}$ in terms of the strength of the evidence against model 2 (Kass \& Raftery 1995; Tan \& Biswas 2012). The rule of thumb is that if $\Delta \lesssim 2$, the evidence is weak; if $\Delta \sim 3-4$, it is adjudged to be mildly strong; and if $\Delta \gtrsim 5$, it is quite strong. As we shall see, in all the cases we consider in this paper, $\Delta \sim$ 4 for each pair of models being compared, so the evidence would be judged 'positive', meaning that it is on the borderline between mildly strong and quite strong.

\section{RESULTS}

We have slightly modified the approach of finding $\gamma$ and $\tilde{\beta}$ from the method used by Risaliti \& Lusso (2019) but, as we shall see, the results are none the less highly consistent with theirs. Here, we have avoided the use of Type Ia SNe as calibrators, which can be difficult to use for model comparisons when various subsamples are merged together to form a large catalogue. Even slight differences in the systematics can cause large effects, as discussed more thoroughly in Wei et al. (2015) and Melia et al. (2018). A purer test of each model using the quasar Hubble diagram bases all of its parameter optimization - those of the model itself, in addition to the unknowns $\gamma, \tilde{\beta}$, and $\delta$ appearing in equations (1)-(5) - on the quasar data alone. This avoids any possible contamination of the calibrators by any unrecognized model dependence. Using this approach, the reduced data themselves will change slightly from model to model, since the inferred luminosities - and therefore the constants $\gamma, \tilde{\beta}$, and $\delta$ - require the use of a model-dependent luminosity distance (equations 6-8). This approach is in fact analogous to what is done with Type Ia SNe, for which the so-called 'nuisance' parameters characterizing the supernova light curve must be optimized along with the free parameters of the cosmology itself, and therefore change slightly from model to model. We use this same approach here with the quasar data, since this appears to be the least biased way of comparing distinct models, such as $R_{\mathrm{h}}=c t$ and $\Lambda \mathrm{CDM}$. 
Table 1. Model comparison based on high- $z$ quasars.

\begin{tabular}{|c|c|c|c|c|c|c|c|c|c|}
\hline Model & $\tilde{\beta}$ & $\gamma$ & $\delta$ & $\Omega_{\mathrm{m}}$ & $a_{2}$ & $a_{3}$ & $\chi_{\mathrm{dof}}^{2}$ & BIC & Likelihood \\
\hline$\Lambda \mathrm{CDM}$ & $6.618 \pm 0.012$ & $0.639 \pm 0.0005$ & $0.231 \pm 0.0004$ & $0.31 \pm 0.05$ & - & - & 1.004 & 1630.26 & 9.79 per cent \\
\hline
\end{tabular}

As we can see in Table 1, we find that the optimized values of the correlation parameters following this method are $\gamma \approx 0.63$ (for the cosmographic fit) and $\approx 0.64$ for both $R_{\mathrm{h}}=c t$ and $\Lambda \mathrm{CDM}$. For the intrinsic dispersion, we find a uniform value of 0.231 in all cases. These are to be compared with the values $\gamma=0.633 \pm 0.002$ and $\delta=$ 0.24 found by Risaliti \& Lusso (2019) using external calibrators. The values of $\tilde{\beta}$ appearing in column 1 of this table correspond to our chosen 'fiducial' value of the Hubble constant, i.e. $70 \mathrm{~km} \mathrm{~s}^{-1} \mathrm{Mpc}^{-1}$ (see the above discussion concerning the linkage between $\beta$ and $H_{0}$ ). And according to equation (3), $\beta$ is then $\sim \tilde{\beta}+0.4$. There are two positive conclusions one may draw from this comparison: (1) the two approaches, of using or not using external calibrators, produce results remarkably consistent with each other, and (2) though the 'nuisance' parameters $\gamma$ and $\delta$ were given the freedom to change from model to model, in the end they appear to be only very weakly dependent on the presumed cosmology, if at all. This lends support to the idea that the correlation assumed in equation (1) does in fact provide a reliable standard candle for model testing, as we have done in this paper.

The different methodology we have employed here does produce a notable departure from the Risaliti \& Lusso (2019) results, however. This is most easily recognized in the optimized values of $a_{2}$ and $a_{3}$ for the cosmographic fit, which we find are closer to those calculated for the standard model by these authors (see fig. 3 in Risaliti \& Lusso 2019) whereas, in their case, the cosmographic fit differed from $\Lambda$ CDM by $\sim \sigma$. Another way to recognize this is via the fact that all three $\chi_{\text {dof }}^{2}$ shown in Table 1 are very close to each other and close to 1, suggesting that all three models fit the data very well. This is easily understood in terms of the internal selfcalibration employed in our analysis. Unlike the use of external calibrators, which essentially fixed the data for Risaliti \& Lusso, in our case the data must be recalibrated for each model, so the optimization procedure enhances the quality of the fit for each cosmology. This is why, in spite of the fact that the three curves in Fig. 9 are not all the same, particularly at high redshift, each fit is adjudged to be very good in terms of $\chi^{2}$, since the data themselves are different for each model.

The simultaneous optimization of the 'nuisance' and model parameters resulted in the correlation shown in Fig. 1 for the $R_{\mathrm{h}}=c t$ universe. The corresponding figures for $\Lambda \mathrm{CDM}$ and the cosmographic fit are so similar to this that there is not much point in showing all three. Though hardly perceptible, the data do change very slightly from plot to plot, reflecting the fact that the nuisance parameters are slightly different from one model to the next (Table 1). In the end, however, the best-fitting correlations are highly consistent among the three models we tested here. To complement the fit in Fig. 1, we also show the residuals corresponding to $R_{\mathrm{h}}=c t, \Lambda \mathrm{CDM}$, and the cosmographic model in Figs 2, 3, and 4 , respectively. The strong similarity between these model fits may also be understood by inspecting the curves in Fig. 9, which shows the optimized distance moduli. The principal difference between these models is that the cosmographic fit deviates slightly from the other two at redshifts beyond $\sim 3$, which, as we shall see, causes a

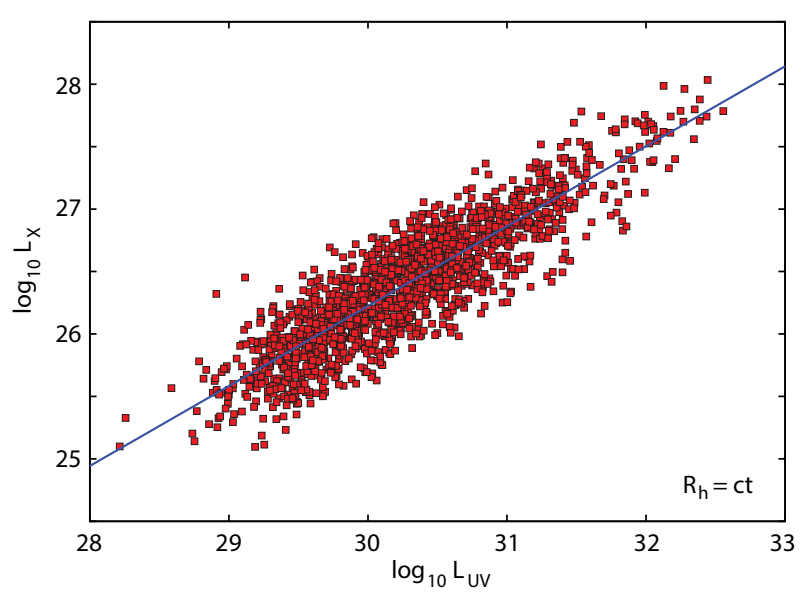

Figure 1. Rest-frame correlation of the monochromatic UV to X-ray luminosity density for the highly selected 1598 quasar sample. The data and fit have been optimized for the $R_{\mathrm{h}}=c t$ model, which has no free parameters. A fiducial value $H_{0}=70 \mathrm{~km} \mathrm{~s}^{-1} \mathrm{Mpc}^{-1}$ has been chosen for the purpose of display only. The actual value of the Hubble constant does not affect the optimization of the key parameters, $\gamma=0.64, \tilde{\beta}=-13.621$, and $\delta=0.231$.

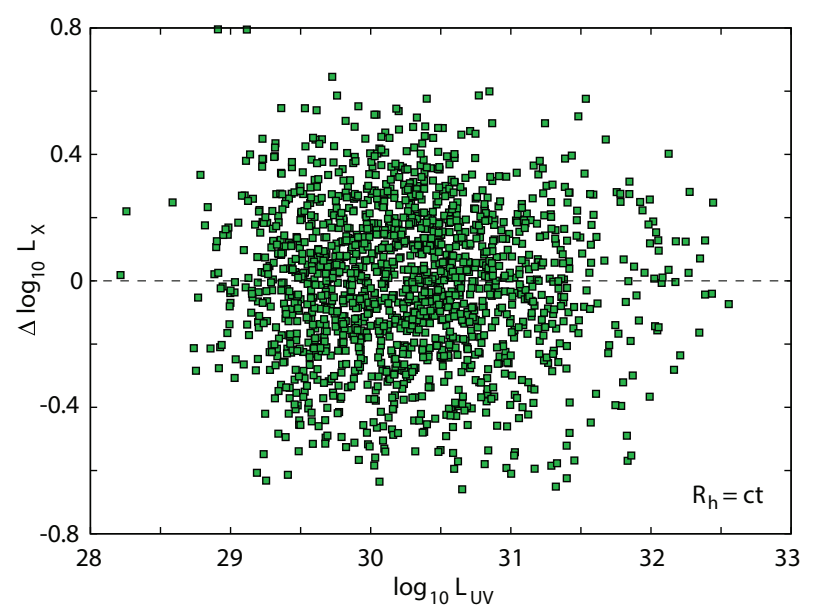

Figure 2. Residuals for the rest-frame monochromatic UV to X-ray luminosity density shown in Fig. 1. The scatter, which is evenly distributed in positive and negative values, is reflected in the inferred intrinsic dispersion $\delta \sim 0.231$ quoted in Table 1 (see also equation 4 and subsequent discussion).

slight bias of the high-redshift Hubble diagram residuals to positive values in this model.

We then used the optimized parameters to calculate the Hubble diagram in each case, which is shown in Fig. 5, again for the $R_{\mathrm{h}}=c t$ universe. The corresponding figures for $\Lambda \mathrm{CDM}$ and the cosmographic model are almost identical, save for slight changes in the recalibrated data. From the Hubble diagrams, we determined the quality of the fits (indicated by the reduced $\chi^{2}$ values quoted in 


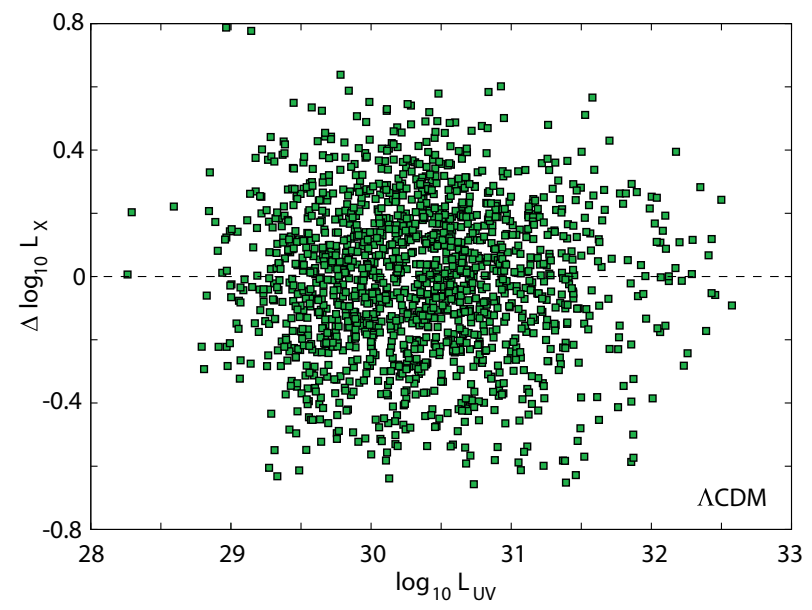

Figure 3. Same as Fig. 2, except now for $\Lambda \mathrm{CDM}$. The scatter in these two models is very similar, so their inferred intrinsic dispersions $\delta$ are identical (see Table 1).

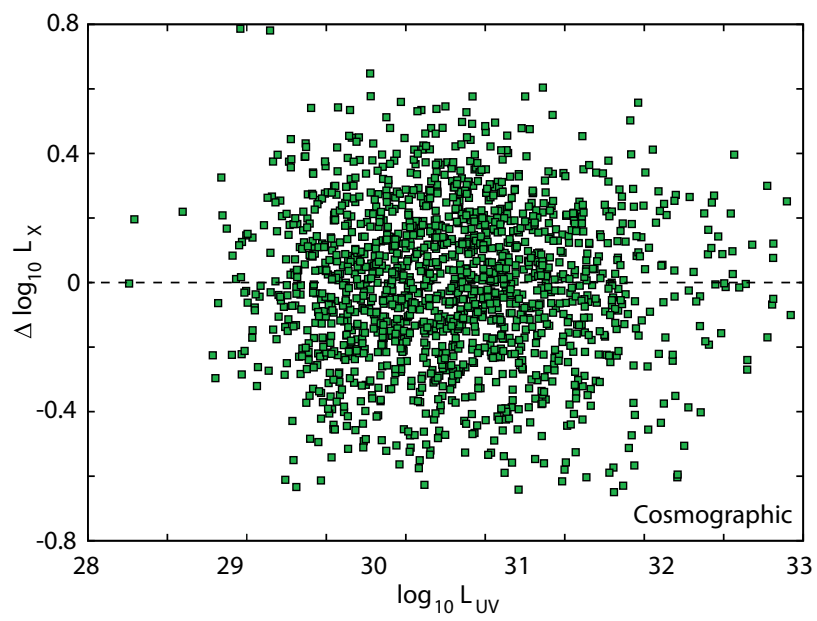

Figure 4. Same as Fig. 3, except now for the cosmographic fit.

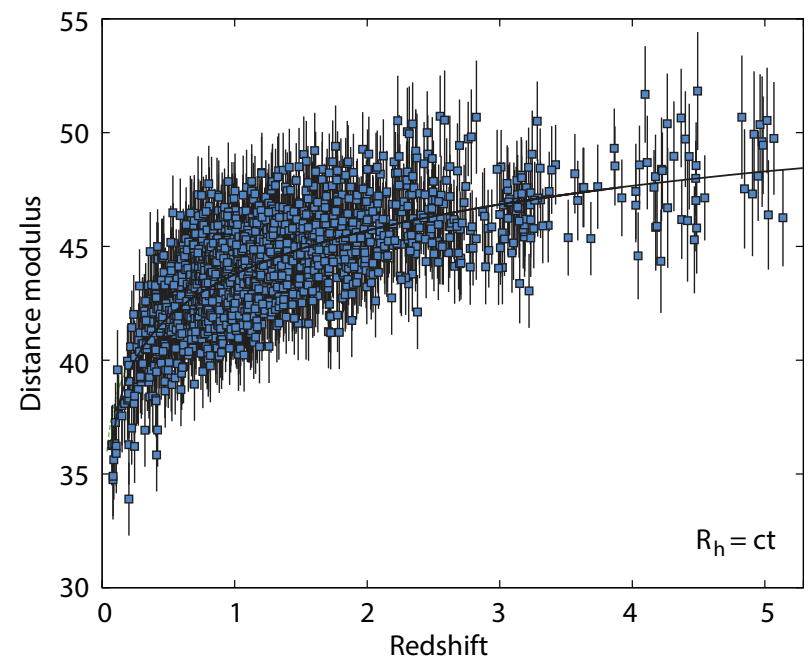

Figure 5. Hubble diagram of the 1598 highly selected quasars for the $R_{\mathrm{h}}=$ ct cosmology. The $\chi_{\text {dif }}^{2}$ for this fit is 1.005 , with a relative BIC likelihood $\sim 88.7$ per cent of this being the correct model.

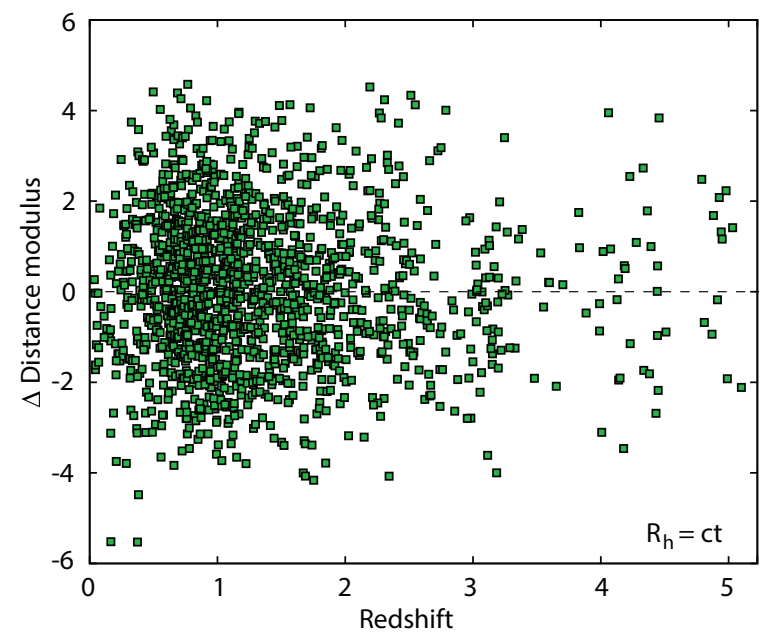

Figure 6. Hubble diagram residuals for the optimized fit shown in Fig. 5.

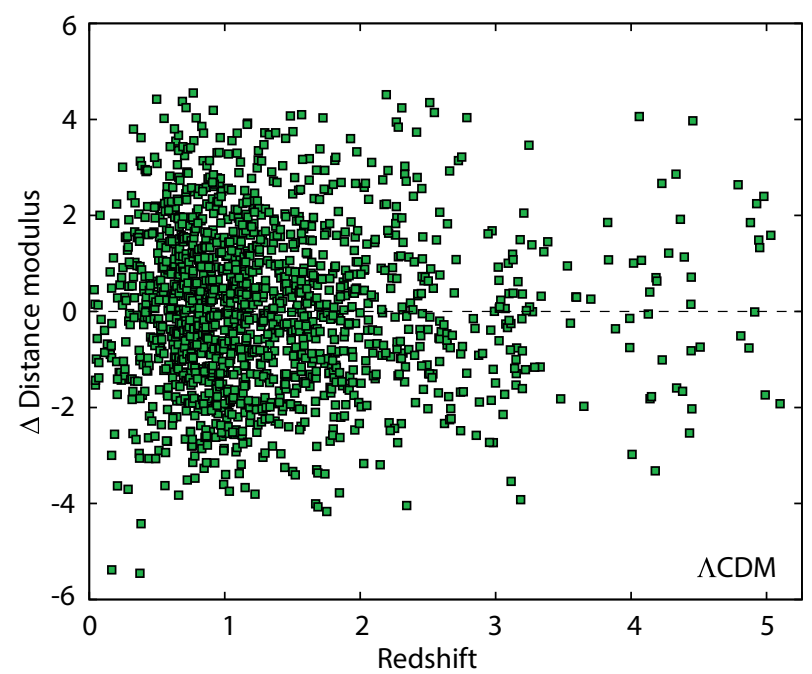

Figure 7. Same as Fig. 6, except now for $\Lambda \mathrm{CDM}$.

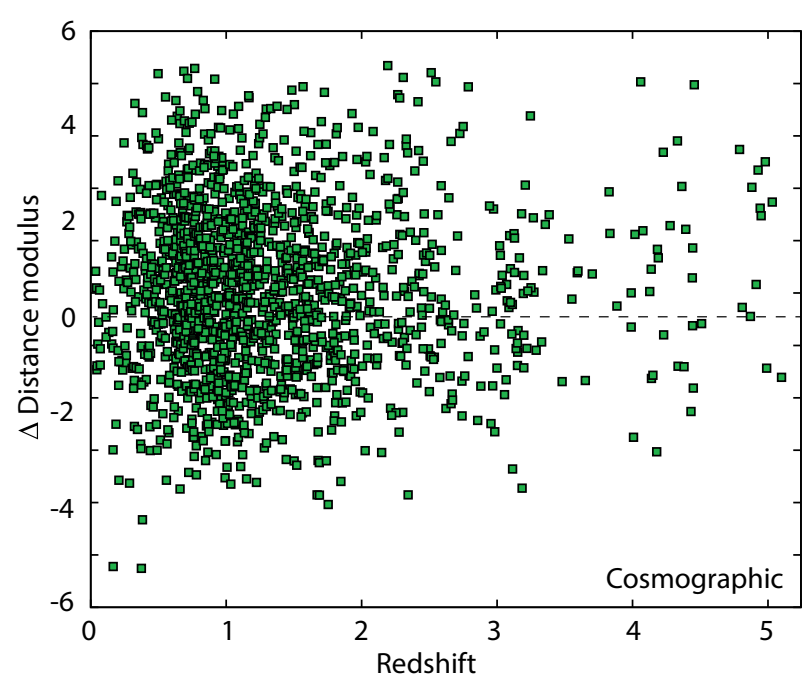

Figure 8. Same as Fig. 6, except now for the cosmographic model. 


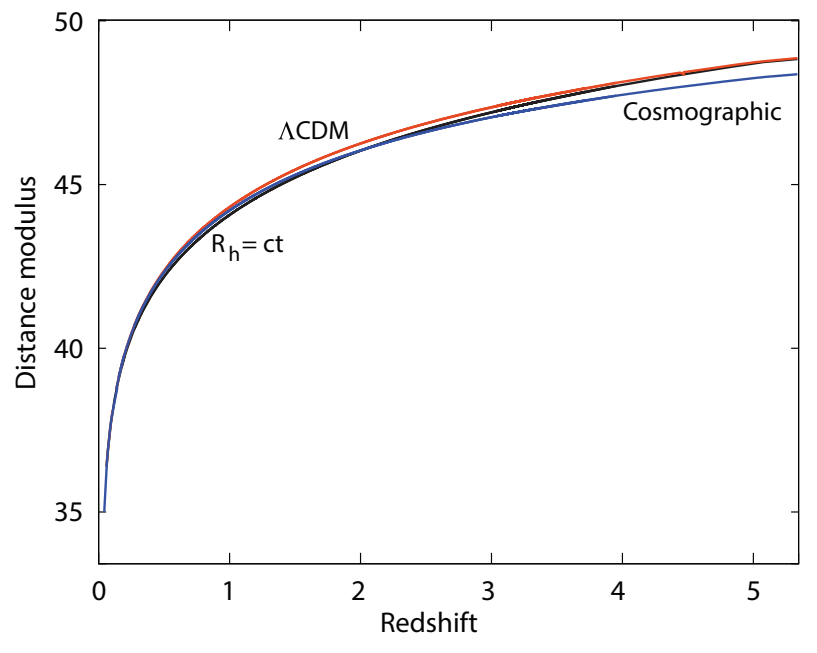

Figure 9. A comparison of all three best-fitting distance moduli, optimized through fits to the 1598 highly selected quasars. The $\Lambda$ CDM curve lies slightly above the other two near $z \sim 2$, while the cosmographic curve is the lowest at $z \gtrsim 3$. The $R_{\mathrm{h}}=c t$ curve corresponds to the fit shown in Fig. 5. Though the cosmographic fit starts to deviate slightly from the other two at redshifts beyond $\sim 3$, the individually recalibrated data also adjust in concert, with the effect that all three reduced $\chi^{2}$ values are virtually identical. The chief difference between these three cases is manifested in the BIC, which also takes into account the number of free parameters used in the optimization procedure.

Table 1) and calculated the BIC likelihoods. Since the sample is so large in this application (specifically $>>30$ ), the BIC is the preferred information criterion to use for model selection (see e.g. Melia \& Maier 2013). As one can see, the reduced $\chi^{2}$ hardly changes from one cosmology to the next, reaffirming the view that this measure of quality is not sufficient to prioritize the models. The cosmographic fit actually has a slightly better $\chi_{\text {dof }}^{2}$ than the other two, but this merely reflects the fact that its greater number of free parameters gives it greater flexibility to adjust to the noise.

To complete the comparison, we also show the Hubble diagram residuals in Figs 6-8. In spite of the overall similarity between these three cosmologies, we do see that, while these residuals are evenly distributed towards positive and negative values for $R_{\mathrm{h}}=$ ct and $\Lambda \mathrm{CDM}$, they are somewhat biased towards positive values at high redshift for the cosmographic model. Together with the comparison of all three distance moduli shown in Fig. 9, in which the cosmographic curve is seen to deviate from the other two at high redshift, we conclude that the cosmographic ansatz is somewhat disfavoured by these data in comparison with both $R_{\mathrm{h}}=c t$ and $\Lambda \mathrm{CDM}$.

The model prioritization may be put on a firmer quantitative basis by considering the BIC likelihoods listed in Table 1. Our main conclusion in this paper is that $R_{\mathrm{h}}=c t$ is preferred by the high- $z$ quasar Hubble diagram in comparison with both the oneparameter, flat $\Lambda \mathrm{CDM}$ cosmology and the empirical cosmographic fit. In Fig. 9 we also learn that $R_{\mathrm{h}}=c t$ and $\Lambda \mathrm{CDM}$ are hardly distinguishable from each other, in spite of the fact that $R_{\mathrm{h}}=c t$ has no free parameters for this fit. The cosmographic fit deviates slightly from the other two at $z \gtrsim 3$, but the impact on the quality of the fits is hardly noticeable. This is due in part to the fact that the data themselves are recalibrated due to the individual optimization of the nuisance variables.

It is clear, therefore, that the differences in the BIC shown in Table 1, and their associated likelihoods, are mostly due to the different number of adjustable parameters among these three models: $R_{\mathrm{h}}=c t$ has none, $\Lambda \mathrm{CDM}$ has one, and the cosmographic fit has two. If we believe that the latter is a reliable, model-independent representation of the data, these results argue in favour of both $\Lambda \mathrm{CDM}$ and $R_{\mathrm{h}}=c t$ being validated by the quasar sample. Indeed, both models fit the data as well as the polynomial expansion in spite of having fewer parameters.

This analysis is also permitting us to see - for the first time how the luminosity distances predicted by $R_{\mathrm{h}}=c t$ and $\Lambda \mathrm{CDM}$ compare with each other over a very large redshift range. It is quite remarkable to see how similar they are over the entire extent of the observations, $0 \lesssim z \lesssim 6$. There is a slight variation at $z \sim$ 2 , but they are virtually indistinguishable elsewhere. This result strongly confirms an indication first discussed in Melia (2015a), that the predictions of $R_{\mathrm{h}}=c t$ seem to act as an 'attractor' for the optimization of model parameters in $\Lambda$ CDM. In other words, the largely empirical parametrization in the standard model appears to account very well for many of the observations because it has sufficient freedom to mimic the much more highly constrained distances in $R_{\mathrm{h}}=c t$.

\section{CONCLUSION}

The outcome of our model comparison using the greatly enhanced high- $z$ quasar sample assembled by Risaliti \& Lusso (2019) has strengthened earlier results based on the quasar Hubble diagram and FSRQ luminosity function, showing that $R_{\mathrm{h}}=c t$ tends to be favoured by these data over $\Lambda$ CDM. Indeed, an extensive compilation of tests such as these (see e.g. table 2 in Melia 2018a) now shows that $R_{\mathrm{h}}=c t$ is a better match than $\Lambda$ CDM to a wide assortment of observations, over a broad range of redshifts. The analysis in this paper is crucial in extending the redshift coverage of these comparative studies to intermediate redshifts $(2 \lesssim z \lesssim 6)$, where few other sources are readily available.

At a redshift $z \sim 6$, the Universe was about 1.9 Gyr old in $R_{\mathrm{h}}=c t$ (and roughly $900 \mathrm{Myr}$ in $\Lambda \mathrm{CDM}$ ), so the work reported here covers a sizeable fraction of the visible Universe. We have found that for much of the evolutionary history in the cosmos, the luminosity distance predicted by the $R_{\mathrm{h}}=c t$ model, which has no free parameters for the work reported here, acts as an 'attractor' for the optimization of model parameters in $\Lambda$ CDM. This follows a trend seen earlier using other kinds of data, including Type Ia $\mathrm{SNe}$ and anisotropies in the cosmic microwave background (Melia 2015a), all of which resulted in optimized fits with $\Lambda \mathrm{CDM}$ that mimicked the predictions in $R_{\mathrm{h}}=c t$, whose tightly constrained observational signatures lack the flexibility of freely adjusting to 'noise' in the data.

An important by-product of our analysis is the affirmation that the correlation seen between the X-ray and UV monochromatic luminosities in these high- $z$ quasars does reliably produce a standard candle for cosmological testing at redshifts not easily accessible by other means, e.g. Type Ia SNe. We have found that the optimization of the 'nuisance' parameters characterizing this correlation depends only weakly on the chosen cosmology. In doing so, we have demonstrated that a reasonable approach in tests such as these is to optimize all of the parameters together - those of the model itself and those in the correlation function - without resorting to external calibrators to fix any of them. One may therefore rely on a highquality sample of quasars such as this for the entire comparative analysis without the concern of introducing possible biases from unrelated data. 
As the $R_{\mathrm{h}}=c t$ cosmology continues to be validated with tests such this, especially towards higher redshifts, its viability strengthens the view that the Universe may actually not have a horizon problem, either in temperature (Melia 2013b), or the electroweak phase transition (Melia 2018b), thereby relinquishing the need of developing a self-consistent inflationary paradigm. Even after four decades of attempts at doing so, we still lack a complete theory for how and when inflation could have taken place. In some sense, one may argue that evidence is now accumulating disfavouring the basic inflationary concept (see e.g. Ijjas, Steinhardt \& Loeb 2013, 2014).

A recent measurement of the minimum cut-off $k_{\min }$ seen in the power spectrum of the cosmic microwave background appears to be incompatible with the basic premise of quantum fluctuations criss-crossing the horizon during an inflationary phase, suggesting that one may not simultaneously solve the horizon problem and the formation of structure in the standard model with the same phase transition (Melia \& López-Corredoira 2018; Liu \& Melia 2019). The work reported here lends some support to the view that inflation may not be needed, and perhaps never happened.

\section{ACKNOWLEDGEMENTS}

I am grateful to the anonymous referees for comments that have led to a significant improvement in the presentation of these results. I am especially grateful to Beta Lusso and Guido Risaliti for sharing their data for this analysis. I am also grateful to the Instituto de Astrofísica de Canarias in Tenerife and to Purple Mountain Observatory in Nanjing, China for their hospitality while part of this research was carried out.

\section{REFERENCES}

Akaike H., 1973, in Petrov B. N., Csaki F., eds, Second Int. Symp. Inf. Theory, Vol. 5, Information Theory and an Extension of the Maximum Likelihood Principle. Akademiai Kiado, Budapest, p. 267

Avni Y., Tananbaum H., 1986, ApJ, 305, 83

Banados E. et al., 2014, AJ, 148, 14

Bentz M. C., Peterson B. M., Netzer H., Pogge R. W., Vestergaard M., 2009, ApJ, 697, 160

Blandford R. D., McKee C. F., 1982, ApJ, 255, 419

Fan X. et al., 2003, AJ, 125, 1649

Ijjas A., Steinhardt P. J., Loeb A., 2013, Phys. Lett. B, 723, 261

Ijjas A., Steinhardt P. J., Loeb A., 2014, Phys. Lett. B, 736, 142

Jiang L., Fan X., Vestergaard M., Jurk J. D., Walter F., Kelly B. C., Strauss M. A., 2007, AJ, 134, 1150

Jones D. O. et al., 2013, ApJ, 768, 166

Just D. W., Brandt W. N., Shemmer O., Steffen A. T., Schneider D. P., Chartas G., Garmire G. P., 2007, ApJ, 665, 1004
Kaspi S., Smith P. S., Netzer H., Maoz D., Jannuzi B. T., Giveon U., 2000, ApJ, 533, 631

Kass R. E., Raftery A. E., 1995, J. Am. Stat. Assoc., 90, 773

Kim A. G., 2011, PASP, 123, 230

Lin H.-N., Li X., Sang Y., 2018, Chin. Phys. C, 42, 095101

Liu J., Melia F., 2019, EPJ-C, in press

López-Corredoira M., Melia F., Lusso E., Risaliti G., 2016, Int. J. Mod. Phys. D, 25, 1650060

Lusso E. A., Risaliti G., 2016, ApJ, 819, 154

Lusso E. A. et al., 2010, A\&A, 512, A34

Melia F., 2003, The Edge of Infinity: Supermassive Black Holes in the Universe. Cambridge Univ. Press, Cambridge

Melia F., 2007, MNRAS, 382, 1917

Melia F., 2013a, ApJ, 764, 72

Melia F., 2013b, A\&A, 553, A76

Melia F., 2014, JCAP, 01, 027

Melia F., 2015a, Ap\&SS, 356, 393

Melia F., 2015b, Ap\&SS, 359, 34

Melia F., 2018a, MNRAS, 481, 4855

Melia F., 2018b, Eur. Phys. J. C, 78, 739

Melia F., López-Corredoira M., 2017, Int. J. Mod. Phys. D, 26, 1750055

Melia F., López-Corredoira M., 2018, A\&A, 610, A87

Melia F., Maier R. S., 2013, MNRAS, 432, 2669

Melia F., McClintock T. M., 2015, Proc. R. Soc. A: Math., Phys. Eng. Sci., 471, 20150449

Melia F., Shevchuk A. S. H., 2012, MNRAS, 419, 2579

Melia F., Wei J.-J., Maier R. S., Wu X., 2018, Europhys. Lett., 123, 59002

Mortlock D. J. et al., 2011, Nature, 474, 616

Paris I. et al., 2017, A\&A, 597, A79

Risaliti G., Lusso E. A., 2015, ApJ, 815, 33

Risaliti G., Lusso E. A., 2019, Nat. Astron., 3, 272

Rosen S. R. et al., 2016, A\&A, 590, A1

Schwarz G., 1978, Ann. Stat., 6, 461

Shafer D. L., 2015, Phys. Rev. D, 91, 103516

Shen Y., Greene J. E., Strauss M. A., Richards G. T., Schneider D. P., 2008, ApJ, 680, 169

Shen Y. et al., 2011, ApJS, 194, 45

Steinhardt C. L., Elvis M., 2010, MNRAS, 406, L1

Tan M. Y. J., Biswas R., 2012, MNRAS, 419, 3292

Volonteri M., Rees M. J., 2006, ApJ, 650, 669

Wandel A., Peterson B., Malkan M., 1999, ApJ, 526, 579

Wei J.-J., Wu X., Melia F., Maier R. S., 2015, AJ, 149, 102

Willott C. J. et al., 2007, AJ, 134, 2435

Willott C. J. et al., 2010, AJ, 140, 546

Yoo J., Miralda-Escudé J., 2004, ApJ, 614, L24

Young M., Risaliti G., Elvis M., 2010, ApJ, 708, 1388

Zeng H., Melia F., Zhang L., 2016, MNRAS, 462, 3094

This paper has been typeset from a $\mathrm{T}_{\mathrm{E}} \mathrm{X} / \mathrm{L}_{\mathrm{E}} \mathrm{T} \mathrm{X}$ file prepared by the author. 